13 Schulze V Jr. Rhabdomyolysis as a cause of acute renal failure. Postgrad Med 1982; 72:145-58.

14 Friedman BI, Libby R. Epstein-Barr virus infection associated with rhabdomyolysis and acute renal failure. Clin Pediatr (Phila) 1986;25:228-9.

15 Zappacosta AR. Myoglobinuria associated with mononucleosis. Journal of American Medical Women Association 1977;32: 428-30.

Accepted 21 October 1988

\section{Rheumatoid nodule formation within the lumbar extradural space}

Sir: The presence of rheumatoid nodules is the major extra-articular feature of rheumatoid disease, occurring typically within the subcutaneous tissues in relation to the extensor surfaces of the wrist and elbow. Rheumatoid nodule formation within the central nervous system is rare. ${ }^{1}$ We report a case of a lumbar extradural rheumatoid nodule giving rise to spinal nerve root compression.

A 58 year old female with a 3 year history of seropositive but quiescent rheumatoid arthritis affecting multiple joints was referred to the Neurosurgical Unit with a 3 month history of left sided sciatica and parathesiae. Examination revealed typical rheumatoid features in both hands but no subcutaneous nodules were seen. There was flattening of the lumbar lordosis and marked restriction of right lateral flexion and extension. Although the left lower limb tendon reflexes were diminished no focal neurological deficit was noted. A lumbar myelogram and CT of the spine revealed a marked stenosis at the L3/4 disc level with a filling defect most prominent in the left postero-lateral position (fig). A mid-lumbar exploration was performed. Exposure of the dura at this level lead to the extrusion of a small amount of pultaceous material. Within the extradural space there was an encapsulated nodule $2 \mathrm{~cm}$ long, lying postero-laterally, and loosely adherent to the dura. The precise origin of the lesion was obscured by fibrous tissue. The mass was removed piecemeal. Histological examination of the lesion showed extensive areas of fibrinoid necrosis, surrounded, in parts, by poorly formed pallisades of histiocytes and chronic inflammatory cells. The appearances were those of a rheumatoid nodule. The patient's sciatica resolved after the operation and she made an uneventful recovery. Her rheumatoid arthritis remained quiescent on penicillamine therapy.
Direct involvement of the brain, spinal cord and meninges by rheumatoid nodule formation is very uncommon. Eleven previous cases of rheumatoid nodule formation within the brain and cerebral meninges have been described,' but only three previous cases of involvement of the spinal cord and spinal meninges. ${ }^{2-4}$ Less than $55 \%$ of the cases of rheumatoid nodule formation in the brain and cerebral meninges had clinical signs or symptoms (and of these a proportion could be attributed to other coexistent CNS pathology.' By comparison all cases with spinal cord involvement (including our case) had neurological symptoms and signs of spinal nerve root compression. This reflects the anatomical site of the lesion rather than any special feature of spinal nodules. Unlike its cerebral counterpart, the diagnosis of spinal rheumatoid nodule does appear to have some therapeutic significance for in two of the three previous cases (as well as in ours) the lesion was readily amenable to surgery. Excision produced resolution of the patients' symptomatology.

Our case is unusual in that it occurred in a patient with inactive disease of only 3 years duration and apart from the extradural

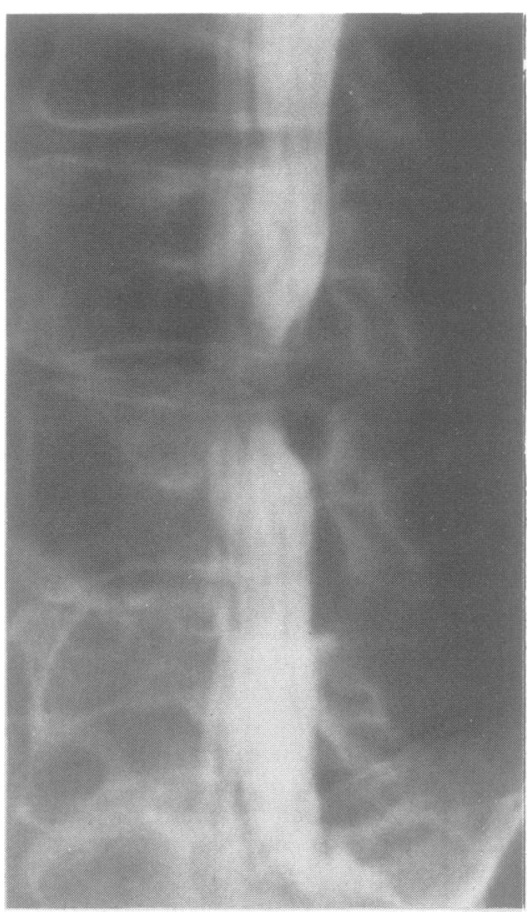

Fig Lumbar myelogram showing a posterolateral filling defect and marked stenosis at the level of the L3/4 disc space. nodule there were no other extra-articular features. All previous examples of CNS으 involvement by rheumatoid nodules have $Z$ occurred in patients with marked synovitis and/or joint deformities. Extra-articular features were also frequent with three patients having ocular involvement, five patients with pulmonary disease and seven ${ }_{\infty}$ with subcutaneous nodules.' Unlike our case, previous examples of CNS rheumatoid $\stackrel{ }{\bar{D}}$. nodule formation appear to be a feature of chronic disease. The duration of disease. varied from 6 to 30 years (although one case $\overrightarrow{\bar{F}}$ with nodules of the falx cerebri and choroid $\stackrel{\oplus}{\rightarrow}$ plexus had only a 4 month history of disease).'

Our case, and the previously reported $\frac{\overline{\bar{\omega}}}{\overline{\mathrm{S}}}$ cases, illustrate the widespread nature of $\overparen{\varnothing}$ rheumatoid disease, but in this case $\mathrm{CNS}_{\mathrm{N}}$ involvement has occurred in the absence of के active disease or other extra-articular $\overrightarrow{0}$ manifestations.

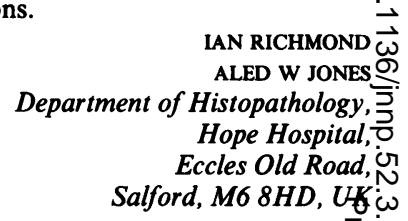
ânt $\frac{A}{A}$

The authors thank RAC Jones, Consultangt $\vec{A}$ Neurosurgeon for allowing them to repoft this case.

\section{References}

1 Jackson CG, Chess RL, Ward JR. A case rheumatoid nodule formation within $\vec{c}$ CNS and a review of the literature. Rheumatol 1984;11:237-40.

2 Gutman L, Hable K. Rheumatoid pachymeningitis. Neurology 1963;13:901-5.

3 Friedman D. Intraspinal rheumatoid nodule causing nerve root compression. $J$ Neurosurg 1970;32:689-91.

4 Markenson JA, McDougal SJ, Tsairis P, et al. $\stackrel{\unrhd}{\circledR}$ Rheumatoid meningitis. A localised immune $\vec{\overrightarrow{ }}$ process. Ann Int Med 1979;90:786-9.

Accepted 14 October 1988

\section{Notice}

The Volvo Award for CNS Injury Research 1989 An award of US $\$ 5,000$ is offered for a paper containing original material, not previously $\frac{D}{O}$ submitted for publication. Details may be obtained from Ass. Prof. Daniel Stålham- N mar, Department of Neurosurgery, Sahlgren Hospital, S-413 45 Göteborg, Sweden. 